\title{
Analyse des pratiques enseignantes et la construction d'un référentiel de compétences : perspectives et contraintes méthodologiques
}

\author{
François Larose, Vincent Grenon, Johanne Bédard et Jimmy Bourque
}

Volume 12, numéro 1, 2009

L'intervention éducative : un cadre conceptuel pertinent

URI : https://id.erudit.org/iderudit/1017488ar

DOI : https://doi.org/10.7202/1017488ar

Aller au sommaire du numéro

\section{Éditeur(s)}

Faculté d'éducation, Université de Sherbrooke

ISSN

1911-8805 (numérique)

Découvrir la revue

Citer cet article

Larose, F., Grenon, V., Bédard, J. \& Bourque, J. (2009). Analyse des pratiques enseignantes et la construction d'un référentiel de compétences : perspectives et contraintes méthodologiques. Nouveaux cahiers de la recherche en éducation, 12(1), 65-82. https://doi.org/10.7202/1017488ar

\section{Résumé de l'article}

L'analyse des pratiques enseignantes oscille présentement entre l'étude des pratiques effectives et l'étude d'un discours sur ces pratiques. Or, les sciences de l'éducation semblent peiner, dans cette entreprise, à quitter le domaine des études dites exploratoires, souvent menées de façon plus ou moins structurée sur un nombre restreint de sujets, défiant ainsi tout effort de généralisation. Cet article présente une démarche scientifique de modélisation des invariants sous-tendant les pratiques. Cette démarche suppose une approche en deux temps : 1) l'étude des représentations de la pratique, formulées par un nombre suffisant de praticiens et dégagées à la suite d'analyses lexicométriques, et 2) l'étude des pratiques effectives, documentées par vidéoscopie et reliées entre elles à l'aide d'analyses factorielles. Des illustrations de la démarche sont fournies. 


\title{
Analyse des pratiques enseignantes et la construction d'un référentiel de compétences: perspectives et contraintes méthodologiques
}

\author{
François Larose, Vincent Grenon, Johanne Bédard \\ Université de Sherbrooke \\ et Jimmy Bourque \\ Université de Moncton
}

\section{Résumé}

L'analyse des pratiques enseignantes oscille présentement entre l'étude des pratiques effectives et l'étude d'un discours sur ces pratiques. Or, les sciences de l'éducation semblent peiner, dans cette entreprise, à quitter le domaine des études dites exploratoires, souvent menées de façon plus ou moins structurée sur un nombre restreint de sujets, défiant ainsi tout effort de généralisation. Cet article présente une démarche scientifique de modélisation des invariants sous-tendant les pratiques. Cette démarche suppose une approche en deux temps: 1) l'étude des représentations de la pratique, formulées par un nombre suffisant de praticiens et dégagées à la suite d'analyses lexicométriques, et 2) l'étude des pratiques effectives, documentées par vidéoscopie et reliées entre elles à l'aide d'analyses factorielles. Des illustrations de la démarche sont fournies.

\section{Abstract}

Teacher practice analysis currently wavers between the study of actual practices and the study of a discourse on these practices. However, in this endeavour, education research seems to struggle to leave the level of exploratory studies, often conducted in a more or less structured fashion with small samples, thus precluding generalization. This paper presents a scientific modeling approach of the underlying invariants regarding practices. This approach is applied in two stages: 1) the study of teacher practice representations as worded by a sufficient number of teachers and uncovered through lexicometric analyses, and 2) the study of actual practices, observed via audiovisual recordings and related to one another with factor analyses. Examples of the approach are given. 


\section{Introduction}

L'analyse des pratiques enseignantes pose une double question. D'une part, elle implique en filigrane la définition plus ou moins formelle de ce que sont ces pratiques. D'autre part, elle soulève la possibilité de développer à moyen et long terme des référentiels de pratique qui soient issus de l'étude des conditions objectives d'exercice de la profession enseignante selon le curriculum en vigueur, l'ordre d'enseignement, le rapport aux savoirs didactisés et aux savoirs de sens commun détenus par les apprenants, etc. ${ }^{1}$

Dans le cadre de cet article, nous aborderons d'abord les fondements d'une logique empirique soutenant le développement et la mise en œuvre d'une démarche scientifique qui permettent de tenir compte de la nature des données issues de l'analyse des observations des conduites enseignantes et de leurs diverses dimensions. En effet, la construction d'une théorie de la pratique implique la recherche d'invariants fondant cette dernière ainsi que, concomitamment, celle des stratégies d'adaptation contextuelles propres aux individus ou aux rapports établis avec les spécificités des élèves ou des tiers acteurs avec lesquels ils interagissent.

Dans ce texte, le terme invariant est utilisé au sens mathématique, soit la présence d'une propriété que divers procédés ne modifient pas. Une propriété concerne un objet conceptuel ou un ensemble d'objets de même ordre. Différentes opérations peuvent être menées pour construire des objets de nature similaire. L'invariance d'une propriété caractérise donc sa stabilité sous ces constructions. La pratique enseignante est un construit (De Landsheere, 1969) ou, si on préfère, une production théorique complexe dont seules les manifestations inférées sont mesurables et observables. En conséquence, l'identification de ces invariants est étroitement associée à la validité de construit qui soutient à la fois la généralisabilité de la théorie et la fidélité des procédures qui en assurent la démonstration.

Dans un deuxième temps, nous présenterons ce qui nous semble être la contrainte majeure permettant d'assurer à la fois la validité des données empiriques et de la théorisation des résultats d'analyse ainsi que celle de la fidélité des mesures permettant d'inférer la présence d'invariants caractérisant les pratiques professionnelles qui structureront un éventuel référentiel professionnel.

En effet, assurer la validité des données et, surtout, celle des inférences issues de leur analyse implique que le recueil de données soit réalisé auprès d'une masse critique de praticiens, sans laquelle la généralisabilité d'une théorie ne peut être assurée. Pour sa part, la fidélité ne peut être affirmée sans que la récurrence des traits caractérisant les pratiques communes aux enseignants ne puisse être vérifiée, donc sans que ne soit réalisée la mesure d'observations répétées chez de mêmes échantillons de praticiens ou auprès d'échantillons comparables. C'est donc la question des critères de scientificité permettant la création d'une théorie de la pratique enseignante qui en permette à la fois une meilleure compréhension et la génération d'hypothèses vérifiables que nous explorerons dans le cadre de cet article. Par la même occasion, nous illustrerons quelques éléments d'une démarche méthodologique tenant compte de la consistance entre données représentationnelles et données «factuelles» qui nous parait prometteuse au regard de l'ébauche d'une méthodologie de l'analyse des pratiques.

1 Dans ce texte, nous utilisons la forme éponyme sans égard à une écriture sexiste, mais pour des fins d'allègement de la syntaxe. 


\section{2. Étude des pratiques effectives des enseignants}

L'étude des pratiques effectives des enseignants fait l'objet d'un intérêt croissant ces dernières années, comme en témoigne la documentation scientifique récente en sciences de l'éducation (Bru et Maurice, 2001 ; Cotton, 1995; Fang, 1996; Jackson, Harper et Jackson, 2001 ; Lenoir, 2005 ; Tupin, 2003). Par ailleurs, cet intérêt se manifeste, peu importe le type de dispositif de soutien à la préparation, à l'actualisation ou à l'évaluation de l'enseignement auquel les praticiens recourent, l'ordre et la discipline d'enseignement ciblée.

D’une manière générale, les recherches portant sur les pratiques enseignantes se fondent en grande partie sur le recueil d'informations de nature indirecte, représentationnelle, fournies par des échantillons plus ou moins consistants de praticiens (Cook, 2002). Concrètement, la majeure partie des écrits traitant des pratiques enseignantes se base sur les résultats d'analyse d'enquêtes par questionnaire, potentiellement réalisées auprès d'échantillons substantiels de sujets. Plus fréquemment, ces écrits se basent sur l'analyse de verbatim d'entrevues plus ou moins structurées, réalisées généralement auprès d'échantillons fort restreints (Clanet, 2005; Suchaut, 2003; Kane, Sandretto et Heath, 2002; Peugh et Enders, 2004). Lorsqu'ils recourent à l'observation des pratiques effectives, soit de façon directe, soit par le truchement de la vidéoscopie, les échantillons sont encore plus restreints et la récurrence des observations est souvent insuffisante pour pouvoir distinguer ce qui relève du caractère invariant des conduites enseignantes de ce qui relève de l'accommodation évènementielle des praticiennes et des praticiens.

Les méthodes d'analyse des données d'observation, peu importe le support sélectionné pour leur recueil, relèvent de logiques éparses prenant alternativement leur source dans les méthodes ethnographiques ou dans les fondements des démarches quantitatives en usage en éthologie humaine ou sociale (Kane, Sandretto et Heath, 2002; Lehner, 2009; Maubant, Lenoir, Routhier, OliveiraAraùjo, Lisée et Hassani, 2005). Bref, ce qui caractérise actuellement l'état de la recherche sur les pratiques enseignantes demeure l'éclatement des méthodes, le caractère représentationnel des données recueillies, ainsi que la faible fréquence de confrontation entre le discours sur la pratique et l'analyse des pratiques effectives. La violation des contraintes caractérisant le recours à certaines méthodes quantitatives et, trop souvent, la surgénéralisation du discours construit à partir de l'analyse de données provenant d'échantillons restreints caractérise aussi beaucoup d'écrits (Bourque, Poulin et Cleaver, 2006; Harwell et Gatti, 2001 ; Larose, Grenon, Bédard, Dezutter, Hasni, Lebrun, Morin, Theis, Thomas et Savoie, 2007; Pearson, 2005 ; Peugh et Enders, 2004).

Ces constats valent d'ailleurs tout autant en ce qui a trait à l'analyse des pratiques en contexte d'enseignement et d'apprentissage «traditionnel» que pour ce qui caractérise l'analyse du recours à divers dispositifs pédagogiques ou technologiques en éducation et en formation. Les plus récentes revues de la documentation scientifique concernant le recours aux TIC en recherche, portant sur l'enseignement, l'apprentissage et, plus particulièrement, sur les pratiques enseignantes en situation de communication médiatisée par ordinateur, arrivent à des conclusions similaires (Luppicini, 2007). 


\section{Les fondements d'une logique empirique: définition d'une démarche scientifique}

L'analyse des pratiques enseignantes implique l'établissement d'un lien logique entre le recours à l'analyse du discours sur la pratique d'une part et, d'autre part, l'observation des pratiques effectives, afin d'en définir les composantes. À son tour, cette démarche implique la recherche d'invariants caractérisant les pratiques de la profession plutôt que les stratégies d'adaptation contextuelle des praticiens. Dans la construction de sa modélisation de l'intelligence, Piaget adopte une posture similaire à ce qui précède dans son usage du concept d'invariance. Donc, comme dans toute démarche de construction de méta-construits hypothétiques telle une théorie de l'intelligence, de la motivation ou de l'apprentissage, la définition d'invariants implique la construction d'une théorie de l'agir enseignant dans laquelle ces invariants s'inscrivent en tant que schèmes organisateurs qui en permettront la généralisation ainsi que l'établissement des limites fonctionnelles.

Un des problèmes majeurs qui se pose lors de la construction d'un programme de recherche portant sur l'identification d'invariants qualifiant la pratique enseignante s'avère la nature même des variables qui seront identifiées. En effet, les sources d'information permettant l'analyse du comportement ou des conduites humaines sont limitées. Elles peuvent se résumer en trois composantes : le discours, la réponse symbolique à l'exposition à un discours et l'observation des comportements. À cet égard, l'observation des conduites enseignantes peut impliquer une séparation ou, au contraire, une intégration de l'analyse des comportements verbaux et non verbaux produits dans le cadre de la situation observée.

Le mode de recueil des données de premier type est généralement l'entrevue, libre, semistructurée ou structurée. Il peut aussi inclure le recours à des dispositifs particuliers tels l'entrevue de groupe ou le groupe focal. Le deuxième type renvoie à l'exposition à tout discours organisé, généralement sous forme de questionnaires ou d'épreuves, imprimés ou sous format électronique. Enfin, le troisième type inclut l'observation directe, plus ou moins critériée, et l'observation indirecte à partir de l'enregistrement des conduites observées sur support audiovisuel.

Les deux premiers types de sources de données impliquent la capacité du sujet à fournir des descripteurs organisés de ses conduites ou encore de prendre position par rapport à des tâches qui décrivent ces dernières ou qui décrivent les manifestations inférées des fondements de celles-ci. Il s'agit donc, dans les deux cas, de données représentationnelles. Les données du troisième ordre peuvent adopter, pour leur part, une configuration de type représentationnel ou objectif. Concrètement, les données issues de l'observation directe sont généralement structurées et organisées de façon plus ou moins formelle lors de leur encodage. La trace ${ }^{2}$ de l'action observée ne correspond donc pas à l'observation elle-même. Elle tend à faire l'effet d'une variabilité incontrôlée associée aux effets de contexte. Inversement, la trace issue de l'enregistrement vidéoscopique des observations est stable. Une fois les intervalles d'observation codés électroniquement, on peut en reprendre les séquences à l'infini pour fin de validation de la grille de codage et de mesure de l'accord interjuges. La trace vidéoscopique peut donc servir de référentiel de validation à l'opération d'encodage qui en permettra la transformation sous forme de données et, dans ce sens, est plus fiable que la première forme.

2 Pour une définition extensive du construit de trace et de ses usages en sciences de l'éducation, le lecteur pourra se référer au premier chapitre de l'ouvrage de Larose et Jaillet (2009). 
Pour résumer notre propos, nous pouvons donc considérer l'existence de trois sources ou de trois formes de données dont deux sont d'ordre strictement représentationnel.

- Les données issues de l'administration d'instruments qui portent sur l'opération que le sujet réalise sur ses propres représentations ou images mentales (données issues d'enquêtes par questionnaire, d'entrevues).

- Les données qui sont le produit d'un encodage direct et qui impliquent un décalage temporel relatif par rapport aux comportements ou conduites observés, comme c'est le cas d'une observation comportementale in situ qui s'appuie sur l'utilisation d'une grille de codage des conduites individuelles ou d'une grille descriptive des interactions sociales comme peut l'être le sociogramme.

- Les données prélevées correspondant directement à la trace (avant encodage de la vidéoscopie) de la conduite observée. Le décalage de la troisième forme de données par rapport aux deux premières se situe essentiellement par rapport à la reproductivité de la situation observée et partant, aux possibilités qui sont offertes au chercheur au plan de la validation de l'instrumentation d'organisation et d'encodage des données.

\section{Essai de modélisation d'une démarche scientifique}

L'exploration de la documentation scientifique portant sur la recherche en sciences humaines et sociales, notamment en éducation, soulève un certain nombre de problèmes. Parmi ces derniers, mentionnons simplement l'existence d'une abondance de recherches qui se présentent sous la bannière exploratoire ou sous une double bannière, celle de démarches qui se veulent à la fois exploratoires et descriptives. Cette tendance est caractérisée par la labilité des fondements et des méthodes de recherche et l'extrême parcimonie des échantillons, allant de l'étude de cas aux échantillons de convenance dépassant rarement la cinquantaine de sujets. Elle ne permet ni la comparabilité des résultats ni même leur additivité rendant à la fois difficile la description de caractéristiques stables des conditions d'exercice de la profession enseignante ainsi que celle des grandes tendances qui qualifient l'enseignement dans divers contextes nationaux (Hargreaves, 1996).

Selon certains auteurs, cette situation serait en bonne partie le produit de la faible importance accordée aux dimensions méthodologiques par plusieurs chercheurs qui œuvrent dans le champ de l'éducation. Cette sous-évaluation les amènerait à masquer derrière un discours se réclamant du qualitatif l'incapacité à tenir compte des démarches permettant la construction d'un échantillon adéquat en fonction de leurs finalités ainsi que de celles qui permettent d'assurer une certaine validité de leurs instruments de recueil de données (Gorard, Rushforth et Taylor, 2004). Gorard (2005) fait d'ailleurs un constat équivalent pour plusieurs chercheurs recourant aux méthodes quantitatives. L'addition des recherches exploratoires qui qualifient le champ serait donc essentiellement le produit d'un manque de culture scientifique chez les chercheurs, associé au manque de rigueur dans l'exploration de l'état de la connaissance scientifique relative aux objets de recherche investigués (Feuer, Towne et Shavelson, 2002). Cette situation rend difficile la construction de méta-analyses ou de revues de la documentation scientifique qui tiennent compte à la fois de la comparabilité des méthodes et de celle des résultats des travaux analysés et qui permettent de prendre en considération la richesse et la diversité des contextes d'exercice de la profession qui sont à l'étude (Bennett, Lubben, Hogarth et Campbell, 2005 ; Duru-Bellat et Merle, 2002 ; Oakley, Gough, Oliver et Thomas, 2005). 
Est-ce à dire que les recherches exploratoires ne sont pas utiles? Certes, non. Elles se justifient lors de la prise en compte de la spécificité d'un contexte local ou régional, d'une méthode ou encore de l'apparition d'un nouvel objet d'étude dans un domaine donné. Néanmoins, elles ne sont que le prétexte et la justification du passage aux étapes ultérieures du déploiement de la recherche, et parmi celles-ci, celui de recherches descriptives dont les résultats comportent un certain degré de généralisabilité. Ces recherches devraient pouvoir tenir compte de la validité interne, donc de la stabilité des données recueillies en tenant compte de la nature de ces dernières. La figure 1 fait état d'une schématisation de la démarche de recherche tenant compte à la fois de la correspondance des échantillons auprès desquels des données portant sur les pratiques enseignantes seront recueillies ainsi que de la complémentarité de ces données.

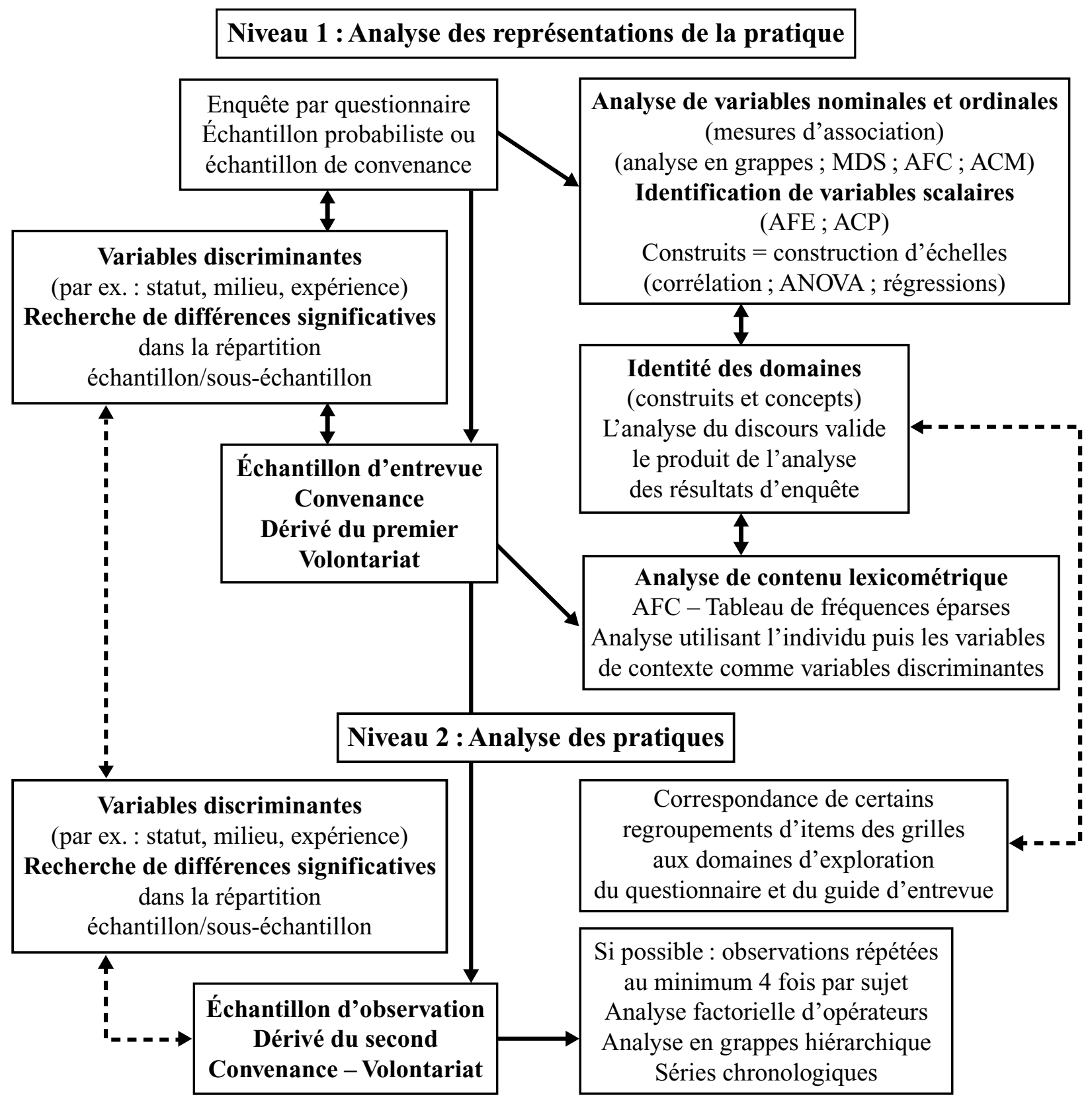

Figure 1 - Démarche de recherche tenant compte de la complémentarité des données, méthodologie schématisée 
Cette démarche de recherche s'inscrit dans une perspective descriptive. Elle assure un certain niveau de validité écologique à la recherche, dans la mesure où elle confronte la stabilité des données représentationnelles recueillies à celles de l'observation en contexte réel (Krathwohl, 1998; Martella, Nelson et Marchand-Martella, 1999). Elle en assure aussi un haut niveau de validité interne, dans la mesure où chaque phase du processus de recueil et d'analyse des données, fait appel à des sources identiques, la stabilité de la répartition échantillonnale en faisant foi. La capacité de projeter la structure de l'échantillon et de ses propriétés sur une population de référence large est, elle aussi, prise en considération.

Nous avons fait état, antécédemment, des limites des données de nature représentationnelle et du besoin de confronter ces dernières à celles qui sont issues de l'observation des enseignants en situation de pratique. Avant d'illustrer la démarche de recherche proposée, il importe donc de définir ce que sont les concepts de représentation et de représentations sociales (RS) et d'établir les fondements d'une conception relativiste de leurs relations avec le réel, par exemple celui de la pratique enseignante.

\section{La relation entre représentations et pratiques}

Pour Kant, les objets de notre connaissance ne sont que des représentations et la connaissance de la réalité ultime est impossible (Dickerson, 2004). Il faudra attendre Durkheim (1898) pour que le concept de représentation acquière ses lettres de noblesse, d'abord en sociologie, puis en psychologie. Pour lui, la représentation recouvre un vaste univers de formes mentales (sciences, religions, mythes, espace, temps, etc.), d'opinions et de savoirs sans distinction. Dans cette perspective, le concept est équivalent à celui d'idée ou de système, ses caractères cognitifs n'étant pas spécifiés. Durkheim recourt d'ailleurs au concept de représentations collectives pour analyser différents phénomènes sociaux en émettant l'hypothèse qu'il est possible d'expliquer ces phénomènes à partir des représentations et des actions qu'elles autorisent chez les individus de différentes catégories sociales. Piaget $(1936,1950,1960)$ introduit une rupture avec les travaux de Durkheim en montrant que si les représentations collectives ont un effet sur les représentations individuelles, l'individu acquiert une autonomie au plan de ses propres représentations au travers de son développement, tant au plan de la socialisation qu'en matière de cognition. Ces représentations évoluent en fonction des rapports successifs qu'il entretient avec la société. Ce faisant, Piaget remet en cause le présupposé durkheimien de l'homogénéité des représentations transmises d'une génération à l'autre au sein d'une collectivité plus ou moins large.

En psychologie, la représentation forme le lien symbolique entre l'environnement extérieur et notre monde mental. Lorsque nous encodons notre expérience du monde, nous construisons des représentations qui vont jouer un rôle essentiel dans notre compréhension et dans nos apprentissages ultérieurs. La représentation forme une synthèse cognitive dotée de qualités de globalité, de cohérence, de constance et de stabilité. Elle est le produit d'un processus de construction individuelle à partir de l'action du réel sur nos sens, de celle des propriétés neuropsychologiques de la mémoire et, enfin, des dimensions affectives ou environnementales, souvent associées au stress, qui agissent tant sur le processus de mémorisation que sur l'organisation et l'activation des schèmes cognitifs qui forment les représentations du réel (Blin, 1997; Vergnaud, 2007). 
En principe, les représentations cognitives ainsi que les schèmes qu'elles forment ont, entre autres fonctions, celle d'orienter nos conduites. Elles sous-tendent donc les attitudes que, à la suite d'Ajzen et Fishbein (1980), reprenant Allport (1935), nous définirons pour les besoins de ce texte comme une variable unidimensionnelle, en l'occurrence, une prédisposition apprise pour répondre à un objet ou à une classe d'objets d'une manière constamment favorable ou défavorable. Dans cette perspective, si l'attitude est stable, la représentation qui la soutient l'est aussi et la conduite probable prévue le sera. Tant la représentation que l'attitude sont des caractéristiques individuelles. Or, on ne peut déterminer d'invariant en recherche à partir de la simple somme de caractéristiques individuelles, surtout lorsqu'elles demeurent de l'ordre du cognitif et de leur médiation en contexte de pratique, donc lorsqu'on désire tenir compte de leur médiation conative.

Les travaux de Moscovici $(1961,2000)$, et surtout les travaux ultérieurs menés par Moliner (1995, 2001), Markova (2003) et Howarth (2006), ont permis d'identifier une dimension spécifique au construit de représentation sociale (RS) qui permet de disposer d'un équivalent collectif aux attitudes. Il s'agit d'une fonction spécifique aux structures périphériques des RS qui permet d'identifier, à l'intérieur d'un corpus de savoirs de sens commun défini en fonction d'un objet social particulier, certaines dimensions prescriptives qui viennent nourrir ou soutenir les attitudes individuelles et, ainsi, permettre d'identifier des prédicteurs probables stables des conduites des membres d'une collectivité. Nous disposons donc ici d'un outil permettant l'identification et la description de certains invariants au plan des schèmes conatifs qui, à leur tour, peuvent contribuer à l'élaboration et à la validation d'une théorie de la pratique enseignante, sous toute réserve du degré de généralisabilité de cette dernière à une catégorie d'enseignants donnée dans un contexte professionnel donné.

\section{Esquisse d'une démarche méthodologique permettant le recueil d'informations portant sur les invariants de la pratique enseignante}

Si l'identification des représentations sociales caractérisant les enseignants d'un ordre donné, dans un contexte social particulier, peut contribuer à documenter leurs pratiques, la mesure de la distance entre leur discours au regard de la pratique d'une part et, d'autre part, leurs pratiques effectives demeurent une démarche incontournable. En effet, les représentations que les enseignantes et les enseignants se font de leurs pratiques et les décisions qui en résultent sont marquées par les biais d'heuristiques décisionnelles, phénomène largement documenté dans divers domaines de l'activité humaine associée à l'organisation des conduites en fonction d'inférences basées sur des données expériencielles (Gilovich, Griffin et Kahneman, 2002). En conséquence, l'objectivation des pratiques enseignantes requiert le recours à l'observation de leurs conduites effectives en classe, leurs communalités en demeurant le critère de prédilection. Nous proposons donc d'illustrer une démarche méthodologique en deux phases permettant à la fois l'identification des composantes stables du discours des enseignants au regard de leurs pratiques, celle de leurs prédicteurs cognitifs et conatifs et la confrontation avec l'analyse de leurs pratiques effectives.

La première consiste à réaliser au moins deux entrevues individuelles avec chaque sujet devant être observé, l'une avant le fait, l'autre en fin d'application du dispositif de recueil de données. La seconde correspond à la réalisation d'une masse critique d'observations des conduites enseignantes et des interactions enseignants-élèves durant des périodes réelles, identiques au regard de l'objet et de finalités de l'enseignement. La valeur minimale de cette masse critique peut être sujette à discussion, mais elle ne devrait pas être inférieure à trois prélèvements par sujet. 


\section{De la stabilité du discours à celle des caractéristiques d'une pratique}

Pour illustrer l'importance de tenir compte à la fois de la stabilité temporelle du discours et celle du rapport entre les données représentationnelles et les données issues de l'analyse de la trace audiovisuelle de la pratique, nous analyserons brièvement quelques informations tirées d'une recherche récemment terminée (Larose et al., 2007). Celle-ci, menée en 2005-2006, visait notamment à identifier les représentations et les profils d'intégration pédagogique du matériel didactique informatisé chez des enseignants du primaire, et à décrire le profil d'intégration de ces matériels par rapport à celui du manuel scolaire à cet ordre d'enseignement. Cette recherche visait à mieux documenter certains éléments d'information relatifs à l'utilisation des TIC en enseignement au Québec, tels qu'identifiés dans le cadre d'une enquête nationale (Larose, Grenon et Palm, 2004) réalisée auprès d'un échantillon stratifié, représentatif, de 1180 praticiens en cohérence avec les résultats obtenus dans le cadre de tierces enquêtes nationales menées dans des pays à systèmes scolaires ainsi qu'à population comparable.

L'enquête faisait ressortir un profil d'utilisation des outils informatiques en tant que matériel didactique, relativement limité. La recherche dont il est fait état ici visait à documenter et à valider le portrait établi auprès d'un échantillon de convenance, restreint, d'enseignants du troisième cycle du primaire qui accepterait concomitamment d'être observés de façon récurrente dans le cadre de périodes de classe régulières. L'échantillon retenu était composé de 13 sujets présentant un profil d'expérience comparable et œuvrant dans des écoles desservant des populations scolaires comparables. La méthodologie impliquait la réalisation de deux entrevues semi-structurées, thématisées; la première était réalisée au début de la troisième étape et la seconde à la fin de la quatrième étape de l'année scolaire. Elle impliquait aussi le prélèvement audiovisuel des interventions de l'enseignant ainsi que de ses interactions avec les élèves durant quatre périodes où il y aurait recours à l'informatique en tant qu'instrumentation pédagogique.

\subsection{Analyse du discours}

Nous avons procédé à l'étude de la distribution des composantes des verbatim en recourant à l'analyse factorielle des correspondances des formes et des segments répétés du discours. Il suffit de mentionner que le principe de base de l'utilisation des modèles factoriels en statistiques textuelles est fort simple. Le discours des individus, en ce qu'il porte sur un objet commun et en ce qu'il se réfère à l'usage d'un vocabulaire contextualisé, implique la présence de concepts stables représentés par le recours à des structures lexicales (mots) et syntaxiques (segments de phrases) récurrentes et communes. Ces mots et ces segments de phrases correspondent théoriquement à une distribution qui devrait respecter les principes de la loi des probabilités conditionnelles de Bayes (Becue-Bertaud et Lebart, 2000). Ils forment le fondement du regroupement des sujets, au regard de leur discours, autour du croisement des axes d'un plan factoriel (barycentres). En fait, ces éléments discursifs constituent le contenu même des dimensions qu'ils partagent au regard d'un concept ou d'un objet symbolique.

L'objectif principal de recourir à cette technique développée par Benzécri (1980) est de représenter à l'aide de graphiques «l'information» contenue dans le tableau des données affiché pour chacune des questions traitées. À partir d'un tableau (n lignes, p colonnes), les données sont représentées par un nuage de points dans un espace de dimension réduite. Ce tableau croise la liste des formes retenues (en ligne) et le code d'identification des sujets (en colonne). Ainsi, dans chaque 
cellule, nous retrouvons le nombre d'occurrences d'une forme dans le discours d'un sujet donné. L'analyse factorielle des correspondances de ce tableau est effectuée par le logiciel en fonction de la fréquence de coupure sélectionnée et de la norme lexicologique énoncée (critère de sélection des formes) pour chacune des questions traitées.

Cette technique, l'analyse factorielle des correspondances, facilite l'identification de la position des indices signifiants du discours puisque le logiciel permet la navigation entre le plan, les formes ou les segments et leur contexte d'utilisation (Grenon, 2000, 2008). Un certain nombre de formes (mots) ou de segments (concepts) caractérisent des individus en particulier. Ces éléments discursifs, en ce qu'ils portent sur des opérateurs (verbes, actions, segments correspondant à des concepts prescriptifs) reflètent la position plus ou moins décentrée d'individus par rapport au centre du plan factoriel. Ces positions décentrées reflètent à leur tour les composantes de variances individuelles résiduelles ou, plus concrètement, la part de variance non expliquée par la prise en considération successive des facteurs. Lorsque l'analyse porte sur des corpus relativement denses de textes provenant d'un petit nombre de sujets, comme c'était le cas lors de l'analyse des verbatim d'entretien des enseignants ayant participé à l'étude, les facteurs de variation par rapport aux barycentres correspondent généralement à une distinction polaire sur le plan des sources de variation individuelle au regard des composantes descriptives ou normatives du discours. Une démarche de ce type est illustrée dans un article de Larose, Jonnaert et Lenoir (1996) portant sur les représentations des didacticiens au regard du concept de didactique et de ses fondements épistémologiques. Pour saisir les données pour analyse statistique textuelle, les auteurs ont eu recours au logiciel Le Sphinx-Lexica. L'utilisation des approches lexicométriques appliquées à l'analyse du discours d'enseignants ou d'élèves a fait l'objet de plusieurs publications que le lecteur pourra consulter afin d'en vérifier la puissance et la pertinence pour notre argumentaire (Larose, Bourque et Lenoir, 2002; Larose, Dirand, Gitzhofer et Bourque, 2006; Pearson, 2005).

\subsection{Analyse des conduites: observations vidéo}

Dans un contexte où la durée, la séquentialité et le nombre d'observations ne permettent pas de recourir aux séries chronologiques dans l'analyse de la trace que constitue l'observation audiovisuelle, on peut utiliser un modèle factoriel particulier: l'analyse factorielle d'opérateurs (AFO). Cette méthode statistique permet d'analyser plusieurs tableaux de données composés des mêmes variables observées sur des individus identiques à différentes occasions (Dazy, Le Barzic, Saporta et Lavallard, 1996).

Les méthodes d'analyse conjointe de plusieurs tableaux de données comportent quatre phases. La première, l'étude de l'interstructure a pour but d'analyser de façon globale les différents tableaux entre eux. On cherche alors à représenter ou à illustrer de manière graphique les tableaux qui se ressemblent et ceux qui sont différents. L'objectif poursuivi dans l'étude de l'interstructure est de déceler des proximités ou des divergences entre les tableaux sans pouvoir donner de description fine des éléments qui les composent. Dans un deuxième temps, le chercheur cherche à identifier un ensemble unique de représentations dont on peut dire qu'il constitue un résumé global, ou encore un compromis de l'ensemble des tableaux. Dans cette étape, le but est de résumer les tableaux de données disponibles en un seul, appelé «compromis», qui doit être représentatif de l'ensemble des tableaux. La troisième étape consiste à l'étude de l'intrastructure ou «analyse fine» des tableaux de données. L'étude de l'intrastructure permet d'analyser les ressemblances ou les différences sur l'aspect des variables mesurées dans les différents tableaux. Finalement, la quatrième étape 
consiste à l'analyse de l'évolution des trajectoires des variables en fonction des différents moments d'observation.

L'étude de l'intrastructure et des trajectoires permet de déceler quelles sont les variables responsables des écarts entre les études ou tableaux correspondant à un moment d'observation chez un ou plusieurs sujets. Il importe ici de préciser que lorsque les différents tableaux étudiés sont indexés dans le temps, on est conduit à décrire l'évolution temporelle d'un phénomène, rejoignant en cela les finalités d'usage des modèles de séries chronologiques (Hamilton, 1994).

Enfin, il importe de spécifier que dans cette méthode, les variables ou les indicateurs se doivent d'apparaitre dans chacun des tableaux pour être retenus. L'absence d'observation d'un indicateur dans un seul des tableaux, donc son absence dans l'une ou l'autre des observations audiovisuelles qui constituent le tableau, nécessite le retrait de cet indicateur et, en conséquence, de la variable qu'il représente, pour l'ensemble des tableaux. La méthode ne permet pas non plus d'analyser des variables pour laquelle la variance est nulle, ces variables prenant alors statut de constantes. Dans la mesure où ce n'est pas l'évolution temporelle d'un phénomène qui nous intéresse, mais plutôt la stabilité de certaines de ces composantes au travers du temps, le recours aux AFO permet d'identifier le caractère stable des composantes d'une empreinte malgré la multiplicité contextuelle des traces.

\section{Quelques résultats illustratifs}

\subsection{Analyse du discours}

Certains items (questions) étaient récurrents dans le cadre des deux entrevues. Ainsi, la question «Dans quel contexte d'enseignement ou lors de quel type d'activités d'apprentissage (projet de groupe, projet individuel, après une séquence d'enseignement d'un contenu, etc.) avez-vous recours à ce matériel? » était présente dans les deux guides, la question étant formulée au passé lors de l'entrevue finale.

L'analyse du discours des 13 protagonistes à cette question fait ressortir un certain nombre d'éléments structurants communs (figure 2).

- Le matériel didactique informatisé est essentiellement utilisé dans le cadre de l'enseignement et de l'apprentissage du français et de la mathématique, ce qui est conforme avec les données recueillies dans le cadre de l'enquête nationale.

- Pour le français, on recourt au travail de groupe autour des projets d'écriture.

- Pour les mathématiques, ce matériel est essentiellement associé au travail individuel.

La comparaison du discours entre le temps 1 (représenté par un a) et le temps 2 (représenté par un b) permet de constater un resserrement de la contribution spécifique de certains sujets autour du discours modal. 


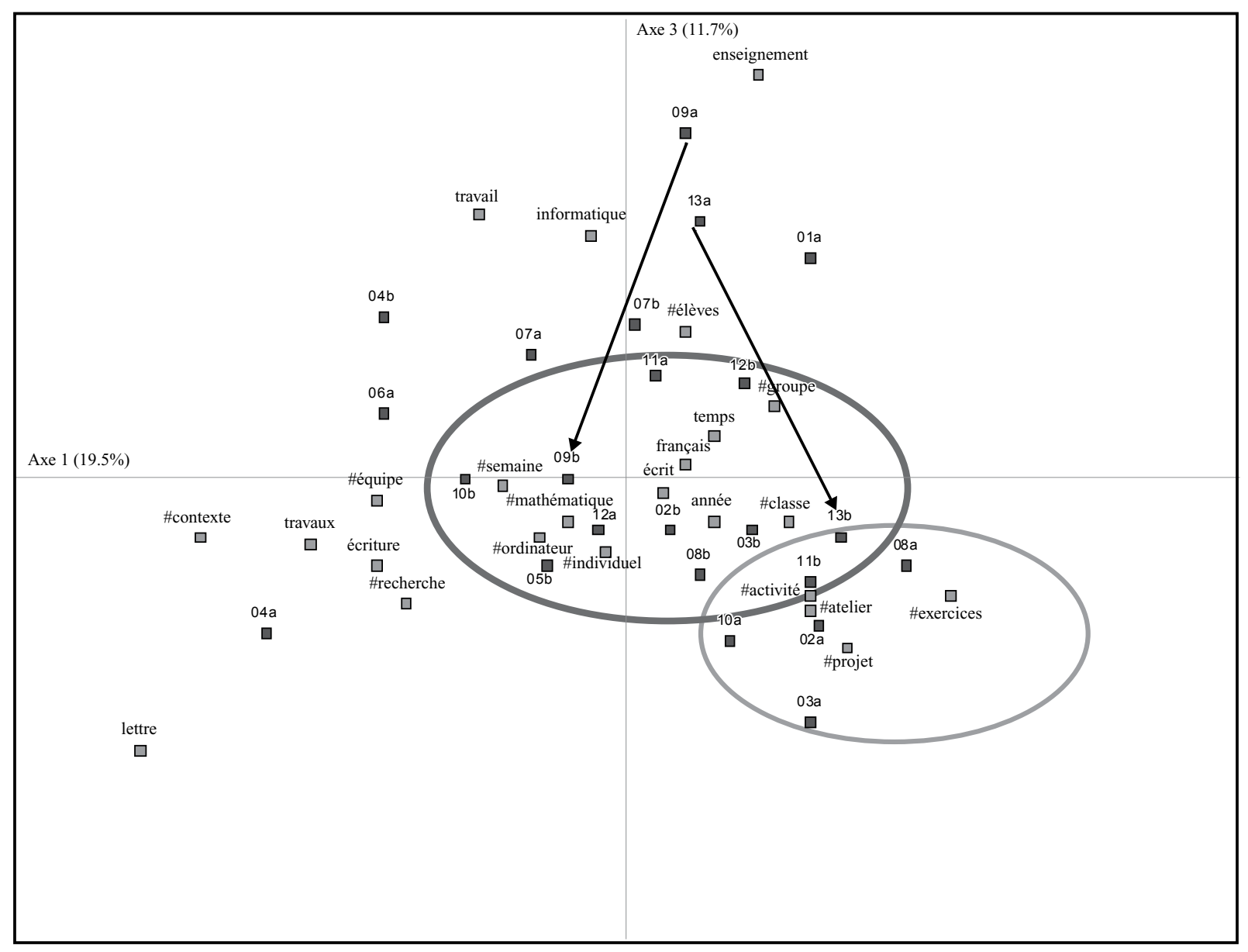

Figure 2 - AFC du discours portant sur le contexte et le type d'activités soutenant la mise en œuvre du matériel didactique informatisé

La dimension exerciseur présente au T1 chez deux sujets s'estompe au T2 et la dimension recours à l'Internet pour fins de recherche, et pour la correspondance scolaire, présente au T1 chez 1 sujet s'estompe au T2.

La variation du discours de certains sujets au travers du temps, en particulier le 09 et le 13 , est importante dans la mesure où elle permet de tenir compte d'un faux effet de variation au plan des composantes représentationnelles à l'étude et qu'elle en permet la neutralisation lors de l'interprétation des données. La correspondance des éléments stables au plan temporel avec les éléments descriptifs obtenus lors de l'enquête nationale vient consolider la validité apparente de ces données.

\subsection{Analyse des prélèvements audiovisuels}

Comme nous l'avons mentionné précédemment, nous disposions de matériel provenant de 52 prélèvements (4 périodes x 13 intervenants). Les périodes observées étaient de durées variables, allant de 50 à 150 minutes, selon l'organisation et la gestion du temps scolaire propre à chaque sujet. Dans la mesure où l'objectif de l'analyse de ces prélèvements était l'identification de tendances stables au plan temporel ainsi que de façon transversale chez les sujets, la variabilité de la durée 
des prélèvements a affecté à la fois le codage des variables permettant d'assurer la comparabilité des données ainsi que la sélection du modèle statistique d'analyse de ces dernières. Nous avons donc opté pour l'identification de 66 variables spécifiques, stables au travers de l'ensemble des bases de données séquentielles produites permettant le recours à l'analyse factorielle d'opérateurs en tant que modélisation de la trajectoire temporelle des conduites des acteurs. L'analyse de données d'observation pertinentes au regard de la question d'entrevue que nous avons préalablement explorée permet un certain nombre de constats (figure 3).

- Les sujets de notre échantillon recourent au MDI (matériel didactique informatisé) essentiellement en laboratoire.

- Le projecteur multimédia, l'accès internet (Navigateur) ainsi que les didacticiels ou exerciseurs sont utilisés par les enseignants en contexte magistral d'abord, puis progressivement dans le cadre du travail en atelier.

- Les enseignants recourent de façon moins systématique à l'internet (figure 3, trajectoires à amplitude maximale) qu'au projecteur multimédia et, en conséquence, au diaporama PowerPoint.

- Certains d'entre eux travaillent fréquemment, mais de façon variable dans les séquences, au développement et à la mise à jour de pages Web.

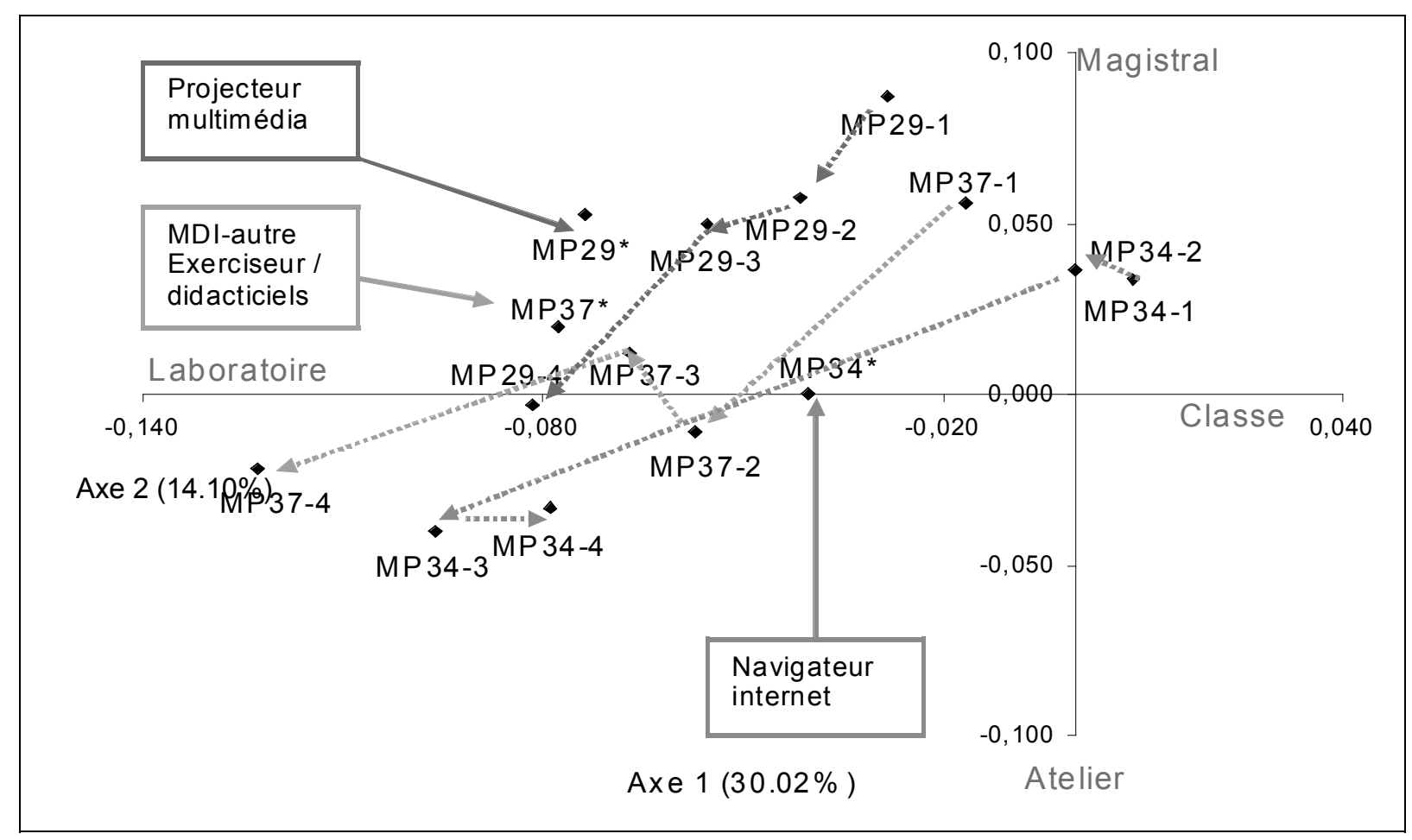

Figure 3 - AFO des pratiques observées (enseignants)

La mise en contexte de ces observations, lorsqu'on tient compte des actions dans lesquelles les élèves sont engagés lors de l'analyse des interactions durant les mêmes périodes, permet de distinguer de façon plus précise les profils de mise en œuvre des TIC ou du MDI. Dans ce cas, nous pouvons constater ce qui suit. 
- Les élèves n'utilisent que peu, à faible fréquence, le projecteur multimédia ainsi que le logiciel de présentation PowerPoint. Ces deux outils demeurent l'apanage de l'enseignant.

- Les élèves n'ont recours à ce matériel que lorsqu'ils présentent les résultats de leurs recherches ou lorsqu'ils doivent expliquer ou solutionner des questions à l'écran.

- On constate une grande stabilité et une fréquence élevée du recours à un didacticiel spécialisé pour le soutien à l'apprentissage du français, le Grammaticiel, dans deux classes. Cette utilisation se réalise en contexte d'atelier, mais à l'instigation de l'enseignant.

- Les élèves recourent de façon fréquente, mais variable dans le temps, aux outils de soutien à l'apprentissage du français (Word) ainsi qu'aux didacticiels autres que le Grammaticiel.

- Certains d'entre eux travaillent fréquemment, mais de façon variable dans les séquences, au développement et à la mise à jour de pages web.

L'intervention des enseignants dans la sélection du MDI mis en œuvre affecte directement le profil d'usage des élèves. En effet, lorsque l'élève sélectionne librement le MDI, il travaille à la création ou à la mise à jour de pages Web ou utilise le Grammaticiel. Lorsque l'enseignant suggère à un élève ou à l'ensemble des élèves d'utiliser un MDI, ceux-ci s'adonnent au courriel, utilisent le Grammaticiel ou un tableur Excel. Enfin, lorsque l'enseignant impose le recours à un MDI aux élèves, ceux-ci sont conviés à une prestation publique (projection PowerPoint), le plus souvent pour illustrer une démarche de résolution de problème en mathématiques (Excel). Pour nos classes cibles, le rapport à l'utilisation ludique de l'informatique est exclu.

\subsection{Comparaison des résultats}

La confrontation de résultats de l'analyse du discours portant sur la question dont nous avons fait état vient confirmer les constats réalisés à la suite de l'analyse des données audiovisuelles, du moins en ce que ces dernières offrent une certaine garantie de stabilité temporelle. Conformément aux résultats obtenus lors de l'enquête nationale, les enseignants recourent peu au MDI pour fins d'enseignement. Lorsqu'ils le font, c'est essentiellement pour fins de présentation et d'animation en classe. Ils utilisent donc essentiellement le projecteur et le diaporama.

Lorsque les élèves sont appelés à utiliser ce matériel pour fins de soutien ou de réalisation d'activités d'apprentissage, ils le font essentiellement au regard de deux matières scolaires, le français et la mathématique et se déplacent (sauf exception de disponibilité d'un didacticiel spécialisé) au laboratoire informatique. Les outils d'apprentissage privilégiés demeurent les outils de bureautique classique (Word, Navigateur internet) et sont mis en œuvre essentiellement dans le cadre de la réalisation de projets d'apprentissage.

Dans un contexte où l'étude des invariants des pratiques effectives et l'identification d'éléments stables ou communs dans le discours des sujets s'avèrent nécessaires au plan de la théorisation de la pratique, le recours complémentaire aux approches lexicométrique et d'analyse factorielle d'opérateurs nous parait une voie à privilégier. 


\section{En guise de conclusion: l'analyse des pratiques enseignantes, un éloge à la complexité}

L'analyse des pratiques enseignantes constitue un processus complexe par rapport auquel une définition préalable de ce que représente le construit de pratique est nécessaire. Il y a là plusieurs postulats théoriques qui sont mis en cause. Jusqu'à quel point le discours sur la pratique que déploient les enseignants reflète-t-il plutôt sa dimension évènementielle ou, au contraire, les déterminants de sa récurrence? Jusqu'à quel point les représentations individuelles reflètent-elles la dimension sociale de l'agir enseignant? Jusqu'où une illustration de pratique contextualisée, telle que reflétée lors d'un prélèvement audiovisuel unique ou encore dans le contexte d'un ensemble disparate d'observations, reflète-t-elle la présence d'invariants caractérisant ou définissant cette pratique? La réponse à cette question implique la mise en place de dispositifs complexes et, avouons-le relativement lourds, mais dont l'absence contribue à maintenir le caractère évènementiel, non généralisable, du discours savant construit autour des enjeux de la définition des déterminants de la pratique enseignante.

La restriction de la plupart des recherches déployées dans ce domaine, dans les pays industrialisés, à des études dites exploratoires, fondées sur l'analyse de fragments de discours d'échantillons restreints de sujets prélevés et étudiés selon des méthodes fort variables, implique, en filigrane, un acte de foi : le discours décrit et prédit les conduites. Au plan scientifique, un tel crédo ne tient pas la route. La même logique s'applique aux pratiques de prélèvements d'échantillons de traces des pratiques enseignantes ainsi qu'à l'éclatement des approches de codification et d'analyse qu'on leur applique. Cet éclatement et le caractère limité du nombre d'observations sur lesquelles on se base pour construire un discours de théorisation de la pratique ne permettent pas de répondre aux questions suivantes.

- Jusqu'à quel point l'observation des conduites enseignantes reflète-t-elle simplement celle de la capacité de l'intervenant à s'ajuster à des contextes spécifiques?

- Jusqu'à quel point l'addition d'études de cas peut-elle correspondre à la description des tendances centrales, stables, caractérisant un échantillon représentatif d'une population donnée et, conséquemment, aux conditions de construction d'un discours qui présente un intérêt hors du champ de l'ethnographie?

Selon le type de réponse à ces questions, le chercheur doit s'inscrire dans une démarche plus ou moins caractérisée par la complexité, dans laquelle les dimensions de complémentarité et de comparabilité qui réfèrent aux concepts de validité et de fidélité en recherche colorent directement la définition qu'on fait du recours aux méthodologies mixtes. C'est dans une perspective de cohérence avec ce que sont ces méthodologies que les éléments de modélisation dont nous avons fait état dans ce texte se situent. Reste à en réaliser les conditions de mise en œuvre dans le cadre d'un programme de recherche cohérent qui définit plutôt la tâche d'une unité de recherche que celle qui contraint la réalisation d'une recherche particulière définie par un objet circonscrit.

Sans le déploiement de telles démarches de recherche inscrites dans une perspective longitudinale, il est illusoire de croire que la formation initiale à l'exercice de la profession enseignante puisse contrecarrer le caractère en apparence surcontextualisé des pratiques des jeunes professionnels en insertion. Ceci notamment à cause de l'influence qu'y exerce le modelage dans le cadre des stages en milieu de pratique. Il est aussi peu probable que, sans la mise en œuvre de telles 
recherches, nous puissions disposer d'informations permettant d'évaluer le rendement réel de l'intervention éducative au regard de l'apprentissage des jeunes, du moins autrement qu'au niveau individuel. Or, à quoi peut donc servir la recherche sur la pratique enseignante si on ne peut mesurer et évaluer l'effet enseignant sur l'objet même de l'exercice de la profession, l'apprentissage scolaire? La systématisation des démarches de recherche permettant l'établissement d'un référentiel de la pratique enseignante demeure donc une condition incontournable des mesures d'impact de l'intervention éducative sur la réussite éducative des jeunes.

\section{Références bibliographiques}

Ajzen, I. et Fishbein, M. (1980). Understanding attitudes and predicting social behavior. Engelwood Cliffs, NJ: Prentice Hall.

Allport, G.W. (1935). Attitudes. In C. Murchison (dir.), Handbook of social psychology (Vol. 2, p. 798-844). Worchester, MA: Clark University.

Becue-Bertaud, M. et Lebart, L. (2000). Analyses statistiques de réponses ouvertes: application à des enquêtes auprès de lycéens. In J. Moreau, P-A. Doudin et P. Cazes (dir.), L'analyse des correspondances et les techniques connexes (p. 59-86). Berlin: Springer-Verlag.

Bennett, J., Lubben, F., Hogarth, S. et Campbell, B. (2005). Systematic reviews of research in science education: Rigour or rigidity? International Journal of Science Education, 27(4), 387-406.

Benzécri, J.-P. (1980). Pratique de l'analyse des données (Vol. 1). Paris: Dunod.

Blin, J.F. (1997). Représentations, pratiques et identités professionnelles. Paris: L'Harmattan.

Bourque, J., Poulin, N. et Cleaver, A. (2006). Évaluation de l'utilisation et de la présentation des résultats d'analyses factorielles et d'analyses en composantes principales en éducation. Revue des sciences de l'éducation, 32(2), 325-344.

Bru, M. et Maurice, J.-J. (dir.) (2001). Les pratiques enseignantes. Contributions plurielles. Les Dossiers des sciences de l'éducation, 5, numéro thématique, 3-117.

Clanet, J. (2005). Contribution à l'étude des pratiques d'enseignement: caractérisation des interactions maîtres-élèves et performances scolaires. Les Dossiers des sciences de l'éducation, 14, 11-28.

Cook, T.D. (2002). Randomized experiments in educational policy research: A critical examination of the reasons the educational evaluation community has offered for not doing them. Educational Evaluation and Policy Analysis, 24(3), 175-199.

Cotton, K. (1995). Effective schooling practices : A research synthesis. Portland, OR: Northwest Regional Educational Laboratory.

Dazy, F., Le Barzic, J.-F., Saporta, G. et Lavallard, F. (1996). L'analyse des données évolutives : méthodes et applications. Paris: Éditions Technip.

De Landsheere, G. (1969). Dictionnaire de l'évaluation et de la recherche en éducation. Paris: Presses universitaires de France.

Dickerson, A. (2004). Kant on representation and objectivity. Cambridge: Cambridge University Press.

Duru-Bellat, M. et Merle, P. (2002). De quelques difficultés à cumuler des savoirs sur les phénomènes éducatifs. L'exemple de la démocratisation de l'enseignement. Revue française de pédagogie, 140, 65-74.

Durkheim, E. (1898). Représentations individuelles et représentations collectives. Revue de métaphysique et de morale, 6. Document téléaccessible à l'adresse <http://classiques.uqac.ca/classiques/Durkheim_emile/Socio_et_philo/ ch_1_representations/representations.pdf>.

Fang, Z. (1996). A review of research on teacher beliefs and practices. Educational Research, 38(1), 47-65.

Feuer, M.-J., Towne, L. et Shavelson, R.-J. (2002). Scientific culture and educational research. Educational Researcher, 31(8), 4-14.

Gilovich, T., Griffin, D.W. et Kahneman, D. (2002). Heuristics and biases: The psychology of intuitive judgement. Cambridge: Cambridge University Press. 
Gorard, S. (2005). Academies as the «future of schooling»: Is this an evidence-based policy? Journal of Education Policy, 20(3), 369-377.

Gorard, S., Rushforth, K. et Taylor, C. (2004). Is there a shortage of quantitative work in education research? Oxford Review of Education, 30(3), 371-395.

Grenon, V. (2000). Méthodes factorielles en statistique textuelle. Application à l'analyse du discours en matière d'informatique scolaire au Québec. Mémoire de maitrise en sciences, Université de Sherbrooke.

Grenon, V. (2008). Impact de la formation en milieu de pratique sur les stagiaires quant au développement de leur niveau d'alphabétisation informatique, de leur sentiment d'auto-efficacité et de leurs attitudes de stress et d'utilité perçue au regard des TIC. Thèse de doctorat en éducation, Université de Sherbrooke.

Hamilton, J.D. (1994). Time series analysis. Princeton, NJ: Princeton University Press.

Hargreaves, D.H. (1996). Teaching as a research-based profession: Possibilities and prospects. London: Teacher Training Agency.

Harwell, M.R. et Gatti, G.G. (2001). Rescaling ordinal data to interval data in educational research. Review of Educational Research, 71(1), 105-131.

Howarth, C. (2006). How social representations of attitudes have informed attitude theories. The consensual and the reified. Theory and Psychology, 16(5), 691-714.

Jackson, R., Harper, K. et Jackson, J. (2001). Effective teaching practices and the barriers limiting their use in accessing the curriculum: A review of recent literature. Peabody, MA: Center for Applied Special Technology, Inc.

Kane, R., Sandretto, S. et Heath, C. (2002). Telling half the story: A critical review of research on the teaching beliefs and practices of University Academics. Review of Educational Research, 72(1), 177-228.

Krathwohl, D.R. (1998). Methods of educational and social science research: An integrated approach (2éd). New York, NY : Addison, Wesley and Longman.

Larose, F., Bourque, J. et Lenoir Y. (2002). Étude des représentations des compétences attendues chez les futurs ingénieurs formés à l'Université de Sherbrooke ainsi que des motifs de sélection de ce programme chez les formés. Rapport de recherche déposé auprès du vice-rectorat à l'enseignement, du vice-rectorat à la recherche et du décanat à la Faculté de génie. Sherbrooke: Université de Sherbrooke, Centre de recherche sur l'intervention éducative (CRIE).

Larose, F., Dirand, J.-M., Gitzhofer, F. et Bourque, J. (2006). Représentation des compétences requises pour l'exercice de la profession chez les formateurs et les élèves ingénieurs à l'Université de Sherbrooke. In B. Fraysse (dir.), Professionnalisation des élèves ingénieurs (p. 203-232). Paris: L'Harmattan.

Larose, F., Grenon, V., Bédard, J., Dezutter, O., Hasni, A., Lebrun, J., Morin, M.-P., Theis, L., Thomas, L. et Savoie, A. (2007). Étude des motifs d'utilisation et des profils d'adoption de matériel scolaire informatisé par des enseignantes et enseignants du primaire au Québec. Rapport final. Sherbrooke/Québec: Université de Sherbrooke, Centre de recherche sur l'intervention éducative (CRIE)/Ministère de l'Éducation, Direction des ressources didactiques.

Larose, F., Grenon, V. et Palm, S.B. (2004). Enquête sur l'état des pratiques d'appropriation et de mise en cuvre des ressources informatiques par les enseignantes et les enseignants du Québec. Résumé administratif: Principaux résultats de l'analyse des questionnaires et des entrevues réalisées. Sherbrooke/Québec: Université de Sherbrooke, Centre de recherche sur l'intervention éducative (CRIE)/Ministère de l'Éducation, Direction des ressources didactiques. Document téléaccessible à l'adresse $<$ http://www.crie.ca/enligne/diffusion.htm> .

Larose, F. et Jaillet, A. (dir.) (2009). Le numérique dans l'enseignement et la formation: analyses, traces et usages. Paris : L'Harmattan.

Larose, F., Jonnaert, P. et Lenoir, Y. (1996). Le concept de didactique : une étude lexicométrique illustrative d'un corpus de définitions. Éduquer et former: théories et pratiques, 8(1), 28-44.

Lehner, P.H. (2009). Handbook of ethological methods ( $2^{\mathrm{e}}$ éd.). Cambridge: Cambridge University Press.

Lenoir, Y. (dir.) (2005). Les pratiques enseignantes: analyse des données empiriques. Les Dossiers des sciences de l'éducation, 14, numéro thématique, 3-112.

Luppicini, R. (2007). Review of computer mediated communication research for education. Instructional Science, $35(2), 141-185$.

Markova, I. (2003). Dialogicality and social representations. Cambridge: Cambridge University Press. 
Martella, R.C., Nelson, R. et Marchand-Martella, N.E. (1999). Research methods: Learning to become a critical research consumer. Needham Heights, MA: Allyn and Bacon.

Maubant, P., Lenoir, Y., Routhier, S., Oliveira-Araùjo, A., Lisée, V. et Hassani, N. (2005). L'analyse des pratiques d'enseignement au primaire: le recours à la vidéoscopie. Les Dossiers des sciences de l'éducation, 14, 61-76.

Moliner, P. (1995). A two-dimensional model of social representations. European Journal of Social Psychology, 25(1), 27-40.

Moliner, P. (2001). Formation et stabilisation des représentations sociales. In P. Moliner (dir.), La dynamique des représentations sociales (p. 15-41). Grenoble: Presses de l'Université de Grenoble.

Moscovici, S. (1961). La psychanalyse, son image et son public. Paris: Presses universitaires de France.

Moscovici, S. (2000). Social representations : Explorations in social psychology. London: Polity Press.

Oakley, A., Gough, D., Oliver, S. et Thomas, J. (2005). The politics of evidence and methodology: Lessons from the EPPI-Centre. Evidence and Policy, 1(1), 5-31.

Pearson, M. (2005). Environnements didactiques informatiques et pédagogie du projet: analyse de l'intervention éducative d'un enseignant en milieu scolaire socioéconomiquement faible. Thèse de doctorat en éducation, Université de Sherbrooke.

Peugh, J.L. et Enders, C.K. (2004). Missing data in educational research: A review of reporting practices and suggestions for improvement. Review of Educational Research, 74(1), 525-556.

Piaget, J. (1936). La naissance de l'intelligence chez l'enfant. Neuchâtel: Delachaux et Niestlé.

Piaget, J. (1950). La construction du réel chez l'enfant. Neuchâtel: Delachaux et Niestlé.

Piaget, J. (1960). La représentation du monde chez l'enfant. Neuchâtel: Delachaux et Niestlé.

Suchaut, B. (2003). De la nécessité d'évaluer les pratiques enseignantes: entre enjeux sociaux et obstacles méthodologiques. Les Dossiers des sciences de l'éducation, 10, 17-30.

Tupin, F. (dir.) (2003). De l'efficacité des pratiques enseignantes? Les Dossiers des sciences de l'éducation, 10, numéro thématique, 2-109.

Vergnaud, G. (2007). Représentation et activité: deux concepts étroitement associés. Recherches en éducation, 4, 9-22. 